\title{
Reflections on Peer Mentoring for Ongoing Professional Development in System Dynamics
}

\author{
George P. Richardson \\ University at Albany \\ gprichardson@albany.edu \\ Laura J. Black \\ Montana State University \\ lblack@montana.edu \\ Michael Deegan \\ US Army Corps of Engineers \\ Michael.A.Deegan@usace.army.mil \\ Navid Ghaffarzadegan \\ Virginia Tech \\ navidg@vt.edu \\ Donald Greer \\ Greer Black Company \\ drgreer@greerblackcompany.com \\ Hyunjung Kim \\ California State University, Chico \\ hkim18@csuchico.edu \\ Luis F. Luna-Reyes \\ University at Albany \\ lluna-reyes@albany.edu \\ Roderick MacDonald \\ Initiative for System Dynamics in the Public Sector \\ rod@isdps.org

\section{Eliot Rich} \\ University at Albany \\ erich2@albany.edu \\ Krystyna A. Stave \\ University of Nevada, Las Vegas \\ krystyna.stave@unlv.edu \\ Nicole Zimmermann \\ University College London \\ n.zimmermann@ucl.ac.uk \\ David F. Andersen \\ University at Albany \\ david.andersen@albany.edu
}




\begin{abstract}
Ongoing professional development in system dynamics can be a challenge. Academics and practitioners often join organizations where they have few close colleagues with the same methodological background. This note describes one long-running effort to address this challenge: a peer mentoring group of system dynamics experts with various degrees of experience and different fields of application that has met weekly during academic semesters over the last four years to discuss their research, works-in-progress, and early ideas. Based on our experience with virtual meetings, we describe the history, motivation and successful practices of the group. Group members reflect on what we value and why we think it works well. We share our experiences not as a prescription for how to run such a group, but in the hopes that they motivate others to initiate and sustain their own peer mentoring groups and share their experiences with the system dynamics community.
\end{abstract}

Keywords: Peer Mentoring, System Dynamics Community, Distributed Learning, Group Processes 


\section{Introduction: Why Peer Mentoring}

A recurring topic in the annual System Dynamics Society Presidential Address is the need to promote high quality system dynamics practice in a way that ensures sustainability and growth of the field. Formal training programs and interaction with colleagues at annual conferences play a critical role in accomplishing these goals but are limited. Most system dynamics graduates do not join departments or organizations with other established senior researchers with similar methodological expertise and therefore do not have opportunities to interact, collaborate, and regularly learn from such colleagues. Without regular opportunities to interact with colleagues familiar with system dynamics, it is often difficult to know whether or how a research idea could make a contribution to the field. As Berger and Luckmann (1966) note, knowledge is socially constructed, so recognizing "a contribution" and "good work" is a social process. A good idea needs a social life; it needs to "go out and meet people."

Mentoring is one approach to organizational learning used in many organizations to connect colleagues across levels of experience and domains of expertise (Bokeno and Gantt, 2000). While mentoring is usually aimed at helping junior researchers, the benefits of peer mentoring go beyond that. Through effective peer mentoring sessions, individuals share their thoughts and ideas, and engage in critical thinking about different problems whether they are about specific research questions or long-term career success. Such meetings help new ideas flourish.

Furthermore, an effective peer mentoring group also benefits senior researchers, keeping them refreshed with new ideas, thoughts, and research trends. Successful peer mentoring processes help the top-down flow of wise experience and bottom-up flow of challenging ideas across individuals in different career stages.

In this note, we introduce our Thursday Group as an example of successful peer mentoring practice. In our case, having a community for ongoing conversations about works-in-progress has been useful to sharpen our thinking and has provided milestones for our ideas to "get out and meet people." In many ways we see what peer mentoring does and how it does it as a rich model for practitioners in pockets around the world to build on. By sharing our experience, we would like to encourage other peer mentoring groups to share their experiences and best practices and promote more researchers and practitioners to engage in peer mentoring in the system dynamics community.

In the following sections, we describe how the group emerged, and we explain the basic goals and structure of the Thursday Group. The last sections constitute a summary of some of our collective reflections on the process. 


\section{Predecessors of the Thursday Group}

The idea of our Thursday Group is not new, but it draws on the experience of two of the founding members of our peer mentoring group, George Richardson and David Andersen. They had first met in 1975 in the System Dynamics Group at MIT. They became members of the Friday Morning Group, a small peer mentoring group whose purpose was to support students in their doctoral work, especially the dissertation-writing process. Much of their prior thoughts about peer mentoring were formed at that point in time.

In 1987, this time at the University at Albany, Andersen and Richardson continued their experience in mentoring at the Decision and Policy Sciences (DAPS) brownbag lunch. This meeting provided an arena where faculty and Ph.D. students who shared an interest in system dynamics and/or the decision sciences met to discuss progress on their research. To maintain momentum, the members met every Thursday even when there was no scheduled topic. Like the original Friday Group at MIT, the DAPS brownbag lunch delighted in being open to half-baked ideas and in helping everyone make progress on their own research agenda. Most members of our Thursday Group participated in the DAPS brownbag lunch at some point in their careers, learning basic values of peer mentoring through the experience and putting them into practice in their own dissertation groups or exporting the practices to their home institutions by initiating a brownbag-like meeting.

By 2011, synchronous-meeting technology had advanced to the point where it was available enough and of sufficient quality to contemplate convening a peer mentoring group of professionals continuing their development at diverse sites around the world.

\section{The Thursday Group}

\section{Who we are}

The first meeting of the Thursday Group started by an email invitation from David Andersen and Laura Black to five other potential participants. The email described the whole purpose of the meeting in the following words:

Our intent is to convene a group of serious scholars in system dynamics who are all currently working on a paper and would, hopefully, welcome group support... In our minds, this group might be something like an on-line version of the DAPS brown bag... When this group gets going, Laura and David envision that each week one of us would make a presentation ... of a current project. The purpose of each meeting would be to help the presenter make progress on his or her work.

At the end of the first meeting on March 3,2011, the group converged on a set of "ground rules" listed in Table 1. As of Spring 2015, the invitation for Thursday Group goes out to 12 members 
on every Wednesday. Our members join the meeting from 4 countries in 5 time zones. The members work in different fields ranging from public policy, public administration, health, business, digital government, informatics, built environment, industrial and systems engineering. Much like a microcosm of the system dynamics community, we are in academia, in research think tanks, and in consulting fields, and we are all in different stages of our professional and academic careers. Our professional interests overlap and intersect; system dynamics is our common language.

Table 1. The Ground Rules of the Thursday Group, developed during the first meeting.

\begin{tabular}{|l|l|}
\hline 1 & The group will meet on Thursdays [11 AM EST] and will be named the Thursday Group \\
\hline 2 & $\begin{array}{l}\text { We will meet regularly every other week, but a member of the group can call for a meeting in the weeks in } \\
\text { between. }\end{array}$ \\
\hline 3 & $\begin{array}{l}\text { We will start on time and end promptly one hour later. It will be considered polite behavior to leave the } \\
\text { meeting promptly to meet another obligation. }\end{array}$ \\
\hline 4 & $\begin{array}{l}\text { We will try to open up the meeting some minutes before the prompt start so that preliminary chatter can } \\
\text { happen and logon problems can get ironed out. Good behavior is to be at the meeting on time or to slip in } \\
\text { quietly, if you are not. }\end{array}$ \\
\hline 5 & $\begin{array}{l}\text { Each session will focus primarily on a research paper being developed for publication by one of the } \\
\text { members. That member will take the lead for that meeting. }\end{array}$ \\
\hline 6 & $\begin{array}{l}\text { All comments will be directed to helping and supporting the person presenting. Pretentious comments are } \\
\text { not allowed. }\end{array}$ \\
\hline 7 & $\begin{array}{l}\text { For each academic semester, a moderator will be selected to keep us all informed over email about who is } \\
\text { presenting next. }\end{array}$ \\
\hline 8 & $\begin{array}{l}\text { Each presentation will have some written document sent out in advance to maximize the value gained in the } \\
\text { online meeting. }\end{array}$ \\
\hline
\end{tabular}

* From the initial grounding rules, rule\#2 was later modified to weekly meetings, and rule\#8 is often honored in the breach.

Besides sharing professional interests, we also share social relationships. The Thursday Group started with some strong relationships among members, and the group has deepened new friendships and re-connected old friends who are now geographically dispersed. Our friendships and peer-mentoring reinforce each other to good effect; sharing humorous moments and a sense of play helps us build a comfort zone for sharing early-stage work and less-polished ideas.

Typically six to eight members participate in the call each week. Not everyone can attend, since we avoid adjusting the meeting date or time to accommodate variations in our teaching, meeting, 
or travel schedules. Rather we strive to generate useful conversations among those present. Sometimes, collaborators outside the Thursday Group are invited if they are involved in the topics presented in the group.

\section{Goals}

The Thursday Group was formed around our shared goal of doing relevant and high-quality system dynamics work and being successful academics and practitioners. The goal for each meeting is to advance the presenter's work by addressing the needs articulated by the presenter. The group's feedback often involves opening up possibilities for research ideas and pointing the presenter to strengths, a fruitful line of argument, publication outlets, and possible collaborators. Presenters can bring work in any stage of progress. We also use sessions to address careeroriented discussions, such as how to take better advantage of a sabbatical or how to manage a job change. The conversations stimulate ideas, and everyone learns from the ideas exchanged in the meetings.

\section{Roles and Processes}

We have three key roles: moderator, presenter, and friendly audience. The moderator manages the logistics of meetings. The presenter brings the meat of the conversation to the meeting, and the audience listens, clarifies, reflects back, and makes suggestions to the presenter. In the following paragraphs, we briefly describe the main processes in which these three roles are involved.

Meeting Preparations. At the beginning of each academic semester, we have a planning meeting. In this meeting, a member would volunteer to serve as the moderator for the semester. With the moderator taking notes, the group collectively creates a presentation schedule. Each member voluntarily picks a date and proposes a topic to be shared. The commitment to present helps us set individual research goals for preparing a conference paper, journal article or grant proposal.

Each week, the moderator sends out a meeting invitation with a link to the online conferencing site and the presentation schedule for the future weeks. This gentle schedule reminder helps us remain aware of our commitments to the group even when our individual work schedule shifts. The invitation can include an abstract or presentation slides distributed by the presenter. Although presentation materials are shared at the time of the meeting via meeting software, distributing the materials ahead of time is helpful as some members may connect only by phone without visual access. For example, while one member was on sabbatical in Ethiopia, she participated via phone while viewing the presenter's slides, which she had downloaded (slowly) the previous day.

Early in our process we settled upon the GoToMeeting ${ }^{\mathrm{TM}}$ platform for our conversations, with Skype $^{\mathrm{TM}}$ as a backup. While these are certainly not the only options, having an established and 
consistent tool reduces the weekly set up time. Both of these tools support video and text chat, shared screen viewing, recordable audio, and file transfer. All of these elements come into play regularly during our sessions. We have also established a cloud-based repository (Dropbox) for sharing presentations, articles, and other artifacts from the meeting.

Typical Meeting Process. We devote one meeting to one presenter or a presenting team. It begins with a friendly chat while people are arriving on the call and we quickly move to the day's topic when it is time to start officially. A meeting is usually one-hour long, and the presenter lets the group know how he or she would like to allocate the time based on the presentation goals. In a typical meeting, a presentation takes about 20 minutes, and it ends with the presenter's specific questions or concerns about the work in process. Because most presentations focus on projects in early to intermediate stages, much of the meeting time is devoted to comments and feedback.

The moderator manages the conversation by keeping track of time and the audience's chat-box comments. The chat box is used to indicate if someone in the audience wants to speak up, make a comment, or suggest a detailed reference. This way we manage to incorporate feedback from everyone, including more reserved participants, while preserving the speaker's continuity and control of the session. When the meeting is over, we follow up by sharing related articles and resources via e-mail.

The moderator also signals the end of the meeting based on the pre-defined schedule. A consistent ending time permits us to schedule around the session with some regularity. While it is not uncommon for meetings to go a few minutes longer, the end signal provides a graceful way for participants to leave and move to their individual activities as needed.

Last Meeting of the Semester. At the end of each semester, we have a meeting celebrating all of our accomplishments in the past months. We follow up on the status of various work presented in the Thursday Group, and we share our other achievements. We also talk about our research goals for the next four to five months.

\section{A Team Reflection on What Has Worked for Us}

While our roles and processes help ensure reliably useful meetings, we believe other aspects also help the Thursday Group work and give it its identity and flavor. An atmosphere of trust for sharing early ideas, our social dynamics, and commitment to mentoring and encouraging together form the foundation. In the following paragraphs we provide our reflections on what we value most as a group and what we believe are the main reasons for the group's success and sustainability. We finish with some suggestions for readers who may want to start a similar group. 


\section{$\underline{\text { Reflections on Peer Mentoring for Ongoing Professional Development in System Dynamics }}$}

\section{What group members value}

First and foremost, we as group members value the opportunity of having regular contact with system dynamics peers from whom we gain professional and collegial "face-to-face" support, even if it is virtual. We build professional confidence through encouragement from colleagues, and offset tendencies toward self-censorship and self-doubt. Colleagues help illuminate the value of a potential contribution with diverse viewpoints, constructive ideas and insights. The meeting provides a space for ongoing learning about our field, and for finding research partners and resources. It motivates and inspires our research.

In the words of group members:

The Thursday Group feels a little like a music Master Class where a young professional gives a short performance for an expert in front of an audience. The expert comments on the performance and sometimes plays or sings a little of the piece too. The audience learns about the craft of music as the expert stops the student at a passage and explains how it might be improved. In the Thursday Group different people are experts about different things. As members comment from their area of expertise, we all learn. The small peer audience keeps the stakes of participation low and mutual respect for each other's contributions to system dynamics keep the motivation for participation high.

The trust among us and our sense of play make this group different from any other setting in which I present. It's a playground.

All of the members seem to genuinely care for the progress and well-being of others in the group. I get a whole lot of very smart people always willing to help me to think through any problem or situation. I think that I get more from than I give to this group.

As individuals, we might self-censor our ideas quite severely. This group encourages their discussion. Sometimes my half-baked ideas get a boost, sometimes they get a boot. But they are always listened to with respect.

Individual satisfaction and growth is an important component of the group. However, beyond the personal satisfaction, the group celebrates the initiation of joint projects, paper publications, conference presentations, professional projects and career advancement (such as tenure cases) inside the group. We all see the Thursday Group as an important factor in many of these achievements. 


\section{Reflections on Peer Mentoring for Ongoing Professional Development in System Dynamics}

\section{Why it works}

Our group has had continued interactions for more than 4 years now, and we believe that the success of our effort comes from several factors, some of them related to our social relationships, and some others related to the process of the group itself.

In the words of group members:

We all have an Albany connection, and overlap in our disciplines. Most of us are in public administration or policy, but others who are in different areas still connect via group model building and organizational topics. So we have a rather close content base. Additionally, the Thursday Group is a social home. I like the people. I look forward to seeing them every week.

I think that it is important that all of the members of this group have known each other well in face-to-face settings before we started to meet on line. So we had a lot of preexistent trust. This is one of the few online meetings where I try to keep my camera turned on. I just enjoy seeing the faces of my friends on a regular basis, and I look forward to the next time that I will see everyone.

It works well because strong collegiality existed prior to the online group and the group maintains it through a casual, supportive, and collaborative culture. [...] There is no pressure to impress others with a polished work.

The social life of this group comes from many different interactions we all have as a common background. However, high commitment and a strong leadership by the two senior members was especially critical in the early stages of the group. It ensured high-quality comments and continues to help other participants in their professional growth.

In terms of process, we believe that our basic principles and rules constitute a key success factor. Using as a name the day that we have committed to meet (Thursday Group) was helpful. It reminded everyone to leave the time slot open for the meeting, and that way, we minimized the schedule conflicts and ensured the continuity of our meeting. Having a moderator for organization and implementing a consistent formal structure would be other examples of effective practices.

Most importantly, we attribute much of the group's success to its casual, supportive, collaborative culture. It allows everyone to appreciate diverse interests and perspectives and learn to take advantage of resources available in the group. Some of our practices reflect this: 
- Focus on the presenter's purpose first

- Use "could's" and "can's" (not "should's" and "ought's")

- Express authentic enthusiasm and engagement for other's ideas

- Hold high aspirations for good work

- Evolve as interests grow or change

We also understand the importance of patience, as it takes time to find opportunities to collaborate and see results. Using individual achievements as collective accomplishments has always been important to create momentum.

\section{Suggestions for Peer-Mentoring Groups and Concluding Remarks}

Based on our experience, we make the following suggestions for those considering the launch of a peer mentoring group.

\section{Meeting Logistics}

- Start with a small core group of people who know and like each other in face-to-face contexts, as this provides a pre-existing social contract that other group members can build on. The International Conference of the System Dynamics Society, the PhD colloquium at the conference, and various local conferences and workshops organized by the Chapters and Special Interest Groups of the society can be places to find like-minded individuals to start such a group.

- Be consistent about meeting times; this reduces overhead for scheduling and coordinating (and for some of us, remembering).

- Use video if possible--the richer communication medium eases many aspects of multipleparticipant conversations.

\section{Interaction Styles}

- Agree explicitly to a few behavioral norms and have a gentle and consistent moderator reinforce them (Everyone takes a turn at being the moderator eventually).

- Have a clear purpose and goals and remind each other of them.

- Enjoy talking with other group members, feel comfortable talking about your work with them, and don't feel pressured to be perfect.

- Strive for quality comments that are rigorous enough to provide value but also be open to flexible and somewhat informal comments.

- Always respect the presenter's goals and intentions. The comments add value if they are useful for the presenter and help the presenter improve his or her work.

- As presenter, know what your questions are so that the group can help.

- Encourage work-in-progress rather than finished work. Applaud risk-takers.

- Take some sessions to discuss future plans/projects and celebrating members successes. 
In this paper, we shared our experience as one model of effective peer mentoring practices. Our note is by no means prescriptive, and we hope more peer mentoring groups come forward and share their own experiences. For example, our Thursday Group members have not yet experienced negative social dynamics or conflict within the group, and therefore, we have not much to add in terms of how to manage conflict within the group. However, if groups who have successfully managed such situations share their experience with the System Dynamics community, all peer mentoring groups will benefit from their experience.

One of us in the Thursday Group sings regularly with a barbershop chorus, and he told us, in the best choruses in the world, every member sings regularly in at least one quartet. That is akin to our hope for peer mentoring: the System Dynamics Society will thrive when each of its members "sings" and harmonizes in a peer-mentoring group.

\section{References}

Berger PL, T Luckmann. 1966. The Social Construction of Reality: a Treatise in the Sociology of Knowledge. Doubleday, Garden City, NY.

Bokeno RM, VW Gantt. 2000. Dialogic Mentoring Core Relationships for Organizational Learning. Management Communication Quarterly 14(2): 237-270. 